\title{
Fast Algorithm for Passive Interference of UHV Transmission Lines
}

\author{
Lu Ying ${ }^{1, a}$, Zhao Zhibin ${ }^{1}$, Zhang Jian gong ${ }^{2}$ and Gan Zheyuan ${ }^{2}$ \\ ${ }^{1}$ College of Electrical and Electronical, North China Electric Power University, Beijing 102206, China \\ ${ }^{2}$ China Electric Power Research Institute, Wuhan, China
}

\begin{abstract}
Passive interference analysis of transmission lines to radio stations is almost entirely based on the method of moments (MOM) or the rapid multipole method and multi-layer fast multipole method (FMM) developed by the method of moments. Based on the above algorithm, this paper proposes to use a diffraction model (UTD) and an optical diffraction method (PO) to model different transmission lines with different radii. The calculation results show that the difference of field strength generated by transmission lines with different radii varies with the distance of the observation point on the transmission line, and the accuracy of the UTO method is much smaller than that of the PO method. It can make good results that provides technical foundation for future modeling.
\end{abstract}

\section{Introduction}

In recent years, with the rapid development of science and technology, massive construction of infrastructure such as transmission lines and radio stations has led to a close distance between the above two ${ }^{[1]}$.In this case, even if the transmission line is not live working, its huge metal structure will affect the reception or transmission of signals from nearby radio stations, which leads to the increasing electromagnetic radiation from transmission lines to radio stations. Therefore the problem that must be considered in power line construction is how to determine the reasonable distance between transmission line and radio station or reduce the passive interference of them.

To solve this problem, the United States ${ }^{[4-6]}$, Canada ${ }^{[7]}$, $\operatorname{Japan}^{[8,9]}$ and other countries have carried out related research on the influence of power lines on signals in different frequency bands such as MF, VHF/UHV, and $\mathrm{m}$ waves since $1960 \mathrm{~s}$, which involves radio wide broadcast ${ }^{[7]}$, radar navigation ${ }^{[5-9]}$ and other fields ${ }^{[8]}$. Since the construction of UHV transmission lines in China, the electric field strength characteristics of UHV transmission lines have a more in-depth research.

According to the working mode and working frequency of radar, the fast multipole method and multilayer fast multipole method(FMM) developed by the moment method are used, and the diffraction model (UTD) and optical diffraction method (PO) are adopted. The passive interference generated by different transmission lines with different radius is solved and analyzed

\section{Modeling}

\subsection{Model establishment}

In the calculation, in order to simulate the influence of the transmission line on the radar operation, it is assumed that the electromagnetic wave direction $(\boldsymbol{k})$ emitted by the radar is perpendicular to the transmission line, the electric field strength $(\boldsymbol{E})$ is parallel to the overhead transmission line, and the electric field strength is $1 \mathrm{~V} / \mathrm{m}(0 \mathrm{dBV} / \mathrm{m})$, the calculation model is shown in Figure 1.

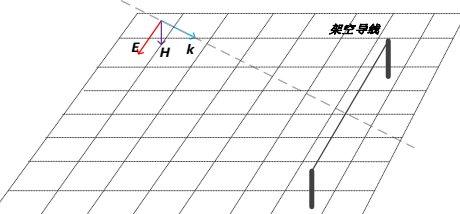

(a) Schematic diagram of the whole model

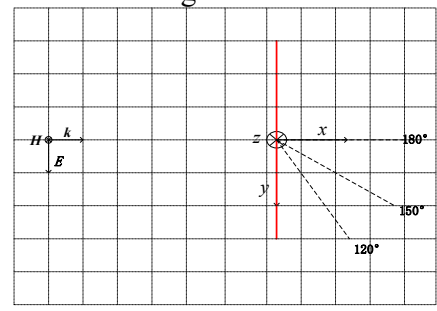

(b) The top view of the model

Fig.1 Passive interference computing model of overhead conductors

\subsection{Frequency selection}

The air-to-air intelligence radar studied in this paper is the commonly used model in China. According to the range from low to high, in the analysis, select 4 frequency points 
of $0.23 \mathrm{GHz}, 1 \mathrm{GHz}, 2 \mathrm{GHz}$ and $4 \mathrm{GHz}$ to calculate.

\section{Influence of wire radius on electric field strength}

In the above modeling case, refereeing to the wire radius of the UHV AC-DC transmission line, the variation law of the electric field strength under the two modeling modes of the transmission line radius of $35 \mathrm{~mm}$ and $45 \mathrm{~mm}$ is calculated respectively.

The other main conditions are as follows:

(1) the length of the wire is $100 \mathrm{~m}$;

(2) calculate the point from $1000 \mathrm{~m}$ away from the wire to $10 \mathrm{~km}$.

The calculation results of passive interference under different wire radius are shown in Figure 2.
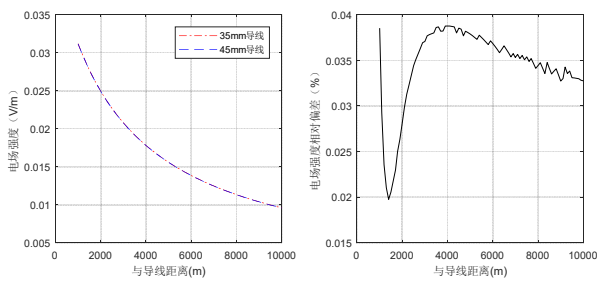

(a)Electric field strength at each point of the vertical line at the frequency of $0.23 \mathrm{GHz}$
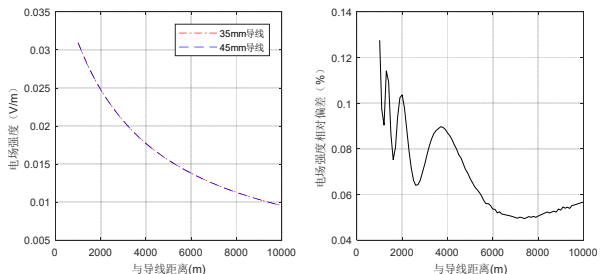

(b)Electric field strength at each point of the vertical line at the frequency of $1 \mathrm{GHz}$
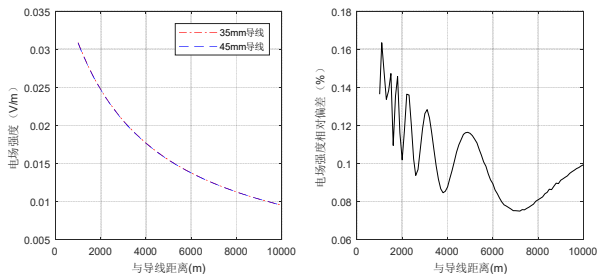

(c)Electric field strength at each point of the vertical line at the frequency of $2 \mathrm{GHz}$
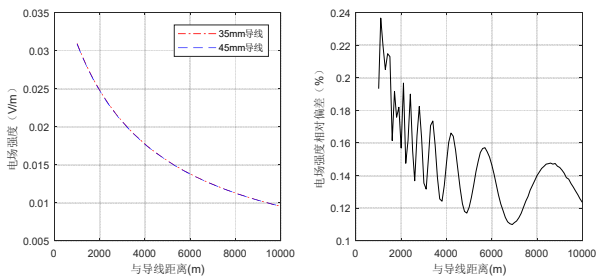

(d)Electric field strength at each point of the vertical line at the frequency of $4 \mathrm{GHz}$

Fig.2 Electric field strength at each point of the vertical line under different radius and different frequencies

From the above figure, we can draw the following conclusions:

Under the same frequency condition, the electric field strength of the transmission line under the different wire radius is basically the same as the distance curve between the observation point and the transmission line, and the deviation of the distance is smaller. According to the trend of the curve, the maximum deviation is less than $0.5 \%$ outside of $1 \mathrm{~km}$.

\section{Comparison of results of different algorithms}

According to the above conclusion that the difference of sub-wire radius has little effect on radar passive interference, and considering the limitations of the computer, this section uses a transmission line with a radius of $35 \mathrm{~mm}$ for calculation.

\subsection{Comparison of calculation results between FMM and UTD}

The passive interference analysis of transmission lines to radio stations is almost entirely based on the method of moments (MOM) or the rapid multipole method and multi-layer fast multipole method (FMM) developed by the method of moments, it is also possible to model calculations using Uniform Diffraction Model (UTD). This section compares the difference between the calculation results of the fast multipole method and the uniform diffraction (UTD). The calculation methods of the cylindrical triangular surface model are FMM and UFO, respectively, and the twisted line model is the reference model of the above comparison.

The main conditions are as follows:

(1)the length of the wire is $100 \mathrm{~m}$;

(2)calculate the point from $100 \mathrm{~m}$ away from the wire to $5 \mathrm{~km}$.

The calculation results of the spatial electric field strength under different calculation methods are shown in Figure 3.
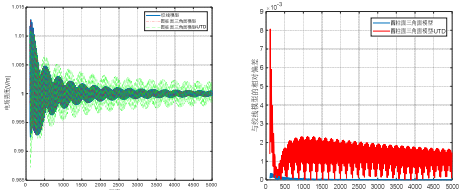

(a)Electric field strength at each point of the vertical line at the frequency of $0.23 \mathrm{GHz}$
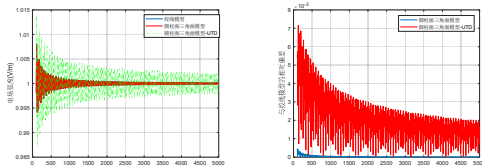

(b)Electric field strength at each point of the vertical line at the frequency of $1 \mathrm{GHz}$
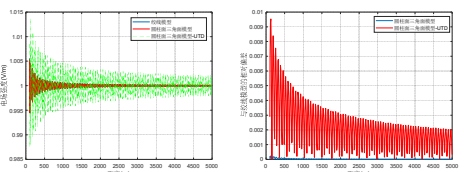

(c)Electric field strength at each point of the vertical line at the frequency of $2 \mathrm{GHz}$ 

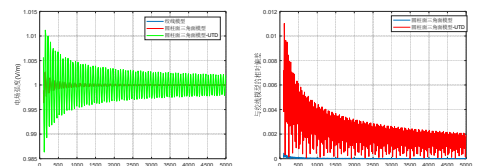

(d)Electric field strength at each point of the vertical line at the frequency of $4 \mathrm{GHz}$

Fig.3 Comparison of calculation results between FMM and UTD

From the above figure, we can draw the following conclusions:

Under the same frequency condition, the electric field strength of the transmission line is basically the same as the distance curve between the observation point and the transmission line under different wire models, and the deviation of the distance is smaller. However, as the frequency increases, the difference in electric field strength obtained by the two calculation methods will gradually increase, and the relative deviation of the electric field strength from the stranded model will also increase. This shows that it is not accurate to calculate the far field of overhead lines by consistent diffraction.

\subsection{Comparison of calculation results between FMM and PO}

Considering the high frequency calculation problem in the calculation of this paper, the optical diffraction method (PO) modeling calculation can also be used. Therefore, this section compares the difference between the calculation results of the fast multipole method and the optical diffraction method (PO), and gives a comparison of the spatial electric field strength from $100 \mathrm{~m}$ to $5000 \mathrm{~m}$.
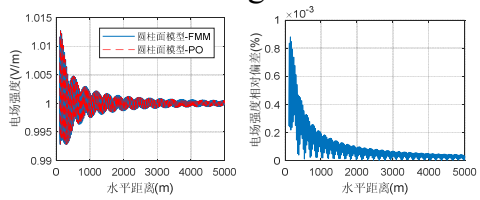

(a)Electric field strength at each point of the vertical line at the frequency of $0.23 \mathrm{GHz}$
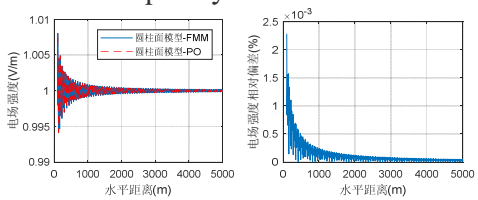

(b)Electric field strength at each point of the vertical line at the frequency of $1 \mathrm{GHz}$
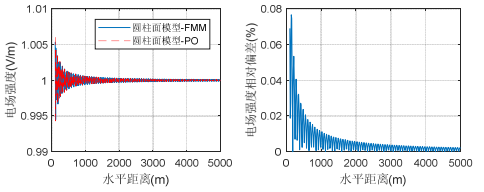

(c)Electric field strength at each point of the vertical line at the frequency of $2 \mathrm{GHz}$
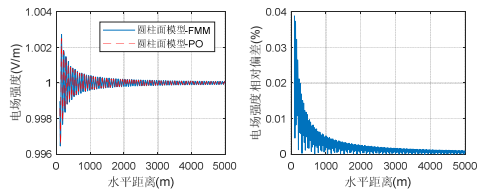

(d)Electric field strength at each point of the vertical line at the frequency of $4 \mathrm{GHz}$

Fig.4 Comparison of calculation results between FFM and $\mathrm{PO}$

From the above figure, we can draw the following conclusions:

Under the same frequency, the electric field strength of the transmission line is basically the same as the trend of the distance between the observation point and the transmission line under different wire models, showing that the deviation of the distance is smaller, and the electric field strength obtained by the two calculation methods is almost the same.

This is consistent with the principle of the physical optical diffraction method that is more suitable for farfield calculations. According to the trend of the curve in the figure, it can be inferred that at any frequency point, the area outside the $1 \mathrm{~km}$, the maximum deviation in value does not exceed $0.02 \%$. This shows that it is accurate to calculate the far field of overhead lines by means of physical optical diffraction.

\section{Conclusion}

Passive interference analysis of transmission lines to radio stations is almost entirely based on the method of moments (MOM) or the rapid multipole method and multi-layer fast multipole method (FMM) developed by the method of moments. Based on the above algorithm, this paper proposes to use a diffraction model (UTD) and an optical diffraction method (PO) to model different transmission lines with different radius. Concluded as follow:

(1)Under the same frequency condition, the electric field strength of the transmission line under the different wire radius is basically the same as the distance curve between the observation point and the transmission line, and the deviation of the distance is smaller. According to the trend of the curve, the maximum deviation is less than $0.5 \%$ outside of $1 \mathrm{~km}$.

(2) In the case of the same wire radius, when the frequency is different, the electric field strength of the transmission line is basically the same as the distance curve between the observation point and the transmission line, and the deviation of the distance is smaller. However, as the frequency increases, the difference in electric field strength obtained by the FMM and UTD will gradually increase, and the relative deviation of the electric field strength from the stranded model will also increase. This shows that it is less accurate to calculate the far field of an overhead line by UTD. In this case, the electric field strength obtained by the FMM and PO is almost the same, and the algorithm principle of the far-field calculation is more consistent with the PO. And at any frequency point, the area outside the $1 \mathrm{~km}$, the maximum deviation in value does not exceed $0.02 \%$. This shows that it is accurate to calculate the far field of the transmission line by means of physical optical diffraction.

\section{Acknowledgement}

This work was supported by the Project of SGCC (GY7116-011). 


\section{References}

1. Yin hui, Zhang xiaoming, Wang yantao, et al. Research on 3D visualization of electromagnetic interference for Ultra-high Voltage transmission lines. High Voltage Engineering.40,12 (2014)

2. Tang bo, Yang jiawei, Huang li, et al. Guard spacing of the air surveillance radar station to Ultrahigh Voltage transmission line.40.4(2018)

3. Tang bo, Sun rui, Zhao xiaoming, et al. Threedimensional Surface Computation model of the Reradiation interference From UHV angle-steel Tower [J]. Proceedings of the CSEE, 31, 4, 2011.

4. Wu xiong, Wan baoquan, Zhang xiaowu, et al. Study on the influence and protection of the radio station between the two circuits of the $1000 \mathrm{kV}$ high voltage exchange [R].Wuhan, Hubei: Wuhan institute of high pressure, 2008.

5. Hill P C.Measurement of reradiation from lattice masts at V.H,F.[J].Proc Inst Elec Eng,111,121964.

6. Kamal Sarabandi, Moonsooo Park. A radar crosssection model for power lines at millimeter-wave frequency[J].IEEE Transactions on Antenna and Propagation, 2003,51(9):2353-2360

7. Truemen C W, Kubina S J. Scatting from power lines with the sky wire insulated from the towers [J].IEEE Transactions on Broadcasting, 1994,40 (2):53-62.

8. Kinase. Theoretical analysis of reradiation characteristics of radio wave caused by transmission lines[ J] .NHK Technology Research , 1968 , 20(3):42-48

9. Takeshita K, Takeshita S, Hashimoto H. Scattering characteristics of $\mathrm{VHF} / \mathrm{UHF}$ television broadcasting waves by overhead owner transmission conductors[ $\mathrm{J}]$. Transactions on Electromagnetic Compatibility,1979,21(1):33-40 